\title{
Desafios para a discussão de missão institucional complexa: o caso de um Instituto de Pesquisa em Saúde
}

\author{
Challenges for the discussion of a complex institutional mission: \\ the case of a health research institute
}

Elizabeth Artmann ${ }^{1}$

M ariaAngélica Carvalho Andrade 2

Francisco J avier U ribe Rivera ${ }^{1}$

${ }^{1}$ Departamento de Administração e Planejamento em Saúde, Escola Nacional de Saúde Pública, Fundação Oswaldo Cruz. Rua Leopoldo Bulhões 1480, M anguinhos 21041-210 Rio de Janeiro RJ. artmann@ensp.fiocruz.br ${ }^{2}$ Hospital Dr. Dório Silva.
Abstract Thisstudy is based on an adaptation of the Strategic Démarche A pproach applied to the Evandro Chagas Institute of Clinical Research (IPEC) in Brazil, from April to July 2009. The results are related to the experience of the Leishmaniasis Laboratory. A strategic analysis of four homogeneous segments was performed, considering the administration of care and specific teaching and research indicators. In both portfolios, all segments showed competitiveness and an appropriate response to mission goals. However, the second portfolio (teaching/research) showed a greater concentration of production in one segment. A highlight was the presence in the work team of cultural traits consistent with the objectives of cultural change. This experience, by operating indirectly in furtherance of the features of the new culture that emerges, revealed the potential of the method in question, to effect policies, processes and resources in a democratic and communicative way, which contributes to the operationalization of the guidelines of the Brazilian Unified Health System.

Key words Participatory management, H ospital management, Cultural management, Organizational change; Health planning
Resumo Este estudo baseia-se na adaptação do método D émarche estratégica no Insti tuto de Pesquisa Clínica Evandro Chagas (IPEC), no período deabril a julho de2009. O sresultados referemse à experiência do Laboratório de Leishmaniose. Foi realizada a análise estratégica de quatro segmentos homogêneos, considerando a produção as sistencial eindicadores específicos de ensino e pesquisa. N o porta-fólio Assi stencial eno deEnsino e Pesquisa todos os segmentos mostraram-se competitivos eadequados à missão. 0 quediferencia 0 segundo éa maior concentração da produção num segmento. Destaca-sea presença na equipedetrabalho de traços culturais coerentes com os objetivos de mudança propostos pel o enfoque. Esta experiência, por operar indiretamente como reforço dos traços da nova cultura que emerge, revel ou o potencial do método em pauta, para efetivar políticas, processos e recursos de forma comunicativa e democrática, o que contribui para a ope racionalização das diretrizes do SUS.

Palavras-chave Gestão participativa, Gestão hospitalar, Gestão cultural, M udança organizacional, Planejamento em saúde 
Introdução

Para enfrentar as dificuldades e os desafios gerados pela implementação do SUS no Brasil, observa-se a emergência de novos modelos de gestão para a efetivação de mudanças nas práticas de saúde. Muitos destes modelos têm limitações para enfrentar mudanças, relacionadas principalmente à resistência das corporações (médicos e enfermeiros), à profissionalização da gestão $0^{1} \mathrm{e}$ à fragmentação e desarticulação das ações de saúdedecorrentes da constituiçãao de equipes do tipo agrupamento ${ }^{2}$.

0 enfoque da Démarche Estratégica de Cré madez e Grateau ${ }^{3}$, desenvolvido no Centro Hospitalar Regional Universitário (CHRU) de Lille/ França propõe-se a promover um processo de transformação cultural progressiva no sentido de apontar para uma maior comunicação entre a gerência estratégica e os centros em organizações do tipo profissional ${ }^{4}$, na busca de um proje to comum que ajude a articular o hospital na rededecuidados. A cultura organizacional éaqui entendida como um conjunto de pressupostos básicos compartilhados, desenvolvidos ao aprender a lidar com os problemas de adaptação externa eintegração internaequefuncionaram bem o suficiente para serem considerados válidos e ensinados aos novos membros como forma correta de perceber, pensar e sentir, em relação a esses problemas ${ }^{5}$. A cultura não évista necessariamente como conjunto homogêneo, mas como processo, como algo em permanente, porém lenta evolução, onde podem conviver elementos de uma "velha" e de uma "nova" cultura. Esta concepção não polarizada da cultura explicaria a presença de elementos aparentemente contraditórios nesta ou uma relativa baixa coerência o que apontaria para uma evolução $0^{5,6}$. U ma situação decrise de paradigmas induz ao aparecimento de novos traços culturais ou de traços emergentes, que viriam superar uma velha identida$\mathrm{de}^{3,6}$. A gestão estratégica pode reforçar indiretamente esses traços emergentes. Os principais impactos culturais almejados ao aplicar a lógica desse enfoque de gestão seriam: favorecer a integração da instituição em uma rede de cuidados estruturada com a preocupação da eficácia e da eficiência; promover uma maior abertura para o ambiente, concretizada pelo reconhecimento e exploração das sinergias e parcerias; atuar deforma multidisciplinar em razão dos esforços de integração com outras especialidadese com a rede; possibilitar a incorporação, pelos profissionais da ponta, de um raciocínio gerencial estratégico, capaz de compensar a diferenciação entre o comando administrativo estratégico e o comando assistencial; modificar o sistema decisional incrementalista para um enfoque de priorização baseado nos custos de oportunidade e em uma visão de conjunto do serviço, que resgate a primazia da solidariedade organizacional; , e propiciar o aumento gradativo da responsabilidade ${ }^{6}$.

As experiências de aplicação desteenfoqueno Brasil analisadas por diversos autores ${ }^{7.14}$, mostram seu potencial como um modelo de gestão estratégica e comunicativa, adequado para abranger a complexa realidade dos hospitais públicos brasileiros ${ }^{7}$.

Consideramos que as instituições de pesquisa - âmbito em que aqui trabalhamos -, compartilham com os hospitais algumas características de natureza organizacional e cultural por serem entidades profissionais4, pela importância que nelas adquire o seu centro operacional. Neste caso específico, a instituição em pauta articula-se a um hospital.

0 objetivo deste estudo foi analisar uma experiência inovadora de aplicação da D émarche Estratégica numa instituição de pesquisa, que adaptou indicadores próprios de pesquisa de forma articulada aos de assistência com o fim de contribuir para consolidar uma metodologia de análise estratégica e de gestão cultural considerando instituições com missões complexas (pesquisa, ensino e assistência).

\section{Método}

Este estudo de caso baseia-se na aplicação do enfoque Démarche Estratégica no Laboratório de Leishmanioseno Instituto de Pesquisa Clínica Evandro Chagas (IPEC) e corresponde aos re sultados parciais de um projeto sobre humanização nos serviços de saúde aprovado nos $\mathrm{Co}$ mitês de Ética da Escola N acional de Saúde Pública e no IPEC.

A escolha da instituição deveu-se ao fato de representar um instituto de pesquisa, assistência e ensino, com reconhecimento nacional e internacional. O IPEC possui a missão de desenvolver pesquisa em Doenças Infecciosas, de caráter multidisciplinar, com base na atenção ao paciente e seu contexto de adoecimento, voltada para a recuperação ea reabilitação do doente, a prevenção da doença e a promoção da saúde. É uma instituição de referência nacional para doenças como Leishmaniose, M icose Sistêmica e D oença Respiratória Aguda Grave, para Diagnóstico His- 
topatológico em Doenças I nfecciosas e credenciado pela Anvisa para realização de ensaios de bioequivalência e biodisponibilidade. Além de desenvolver pesquisas e atividades assistenciais, possui um Programa de Pós-Graduação stricto sensu em Pesquisa Clínica de D oenças Infecciosas (M estrado e Doutorado) e cursos lato sensu, de especialização, residência médica e de capacitação, contribuindo para o aperfeiçoamento de recursos humanos do SUS.

0 projeto foi desenvolvido nos Laboratórios de pesquisa cuja escolha obedeceu ao critério de maior representatividade em termos de produção assistencial ede pesquisa na instituição ecom o aval da direção estratégica da instituição que participou da escolha. Este estudo limita-se a apresentação da aplicação da Démarche no Laboratório de Leishmaniose.

A experiência neste Laboratório envolveu toda a equipe (chefia, profissionais de saúde e estudantes) em reuniões/oficinas de trabalho realizados no período de abril a julho de 2009.

Foram apresentadas as bases conceituais do enfoque metodológico, distribuindo-se material informativo para os participantes. Para a fase 1 , Análise do Existente, foram realizadas reuniões com profissionais da Coordenação de Pesquisa para o levantamento da produção científica (pesquisas em andamento e pesquisadores) e com as chefias do Serviço de Estatística e D ocumentação e do Centro Hospitalar para o levantamento da produção assistencial. Ressalta-se a boa organização do sistema de informação da instituição. Fez-se um levantamento de dados do contexto e dados epidemiológicos esocioeconômicos da região onde o instituto se insere; da relação de patologias e detecnologias por especialidade, ordenadas de acordo com a complexidade; do cruzamento entre patologias etecnologias, identificando a articulação entre tecnologias epatologias ea relação das modalidades de atenção. Esta fase incluiu ainda o levantamento da relação dos concorrentes/parceiros, sendo que a maioria encontra-se em outros estados.

Além desta fase que corresponde aos diagnósticos administrativo e médico e ao levantamento dos dados de produção em pesquisa e inclui a listagem de parceiros e concorrentes institucionais ${ }^{13}$; consideraram-se: Fase 2) Segmentação: consistiu na definição coletiva dos principais agrupamentos homogêneos de atividades a partir de quatro critérios que se aplicam de maneira conjugada: patologia, tecnologia, modos de atenção e população ${ }^{15}$; Fase 3) Análise do valor e da posição competitiva de cada segmento resul- tante dos agrupamentos deatividades: essas duas fases pressupõem um amplo compartilhamento de informações e experiências entre os atores envolvidos. 0 valor refere-se ao interesse relativo ou ao nível de prioridade relativo que os segmentos teriam para o laboratório/instituição, em função de uma política geral, e se expressa pelo interesse em thes alocar recursos ${ }^{12}$. A análise do valor édemulticritérios, incluindo, por exemplo, a perspectiva de crescimento do segmento, o nível de concorrência enfrentado, o nível de investimento tecnológico a ser realizado, etc. $\mathrm{Na}$ análise do valor, a definição do peso relativo desses critérios de avaliação foi obtida distribuindo-se 100 (cem) pontos entre os critérios, em função de sua importância relativa para cada segmento. Posteriormente foi efetuada a análise e a hierarquização de cada segmento, a partir de atribuição de notas de 0 a 20, segundo critérios relativos. O valor final de cada segmento é obtido por um score resultante do produto dos respectivos pesos pelas notas. A posição competitiva foi avaliada considerando-se o grau de controle sobre os chamados Fatores-Chave de Sucesso (FCS), que corresponderiam a vantagens controladas para a garantia de êxito em uma atividade ${ }^{13}$. A avaliação da posição competitiva supõe, em primeiro lugar, a listagem dos FCS. Em seguida, definiu-se o peso relativo desses FCS, distribuindo-se 100 pontos entre os mesmos, em função de sua importância relativa. Posteriormente foi efetuada a atribuição de notas de 0 a 20 a cada FCS em função do grau decontrolereal dos mesmos, tal qual na atribuição de valores aos segmentos. 0 produto dos pesos dos FCS pela nota respectiva definiu scores parciais, cuja soma correspondeao score da posição competitiva decada segmento. $E$, finalmente, determinou-se a posição relativa dos segmentos e a comparação dos scores com os concorrentes/parceiros. Por concorrenteentende-seaqui um mesmo tipo de oferta sediada em outra instituição ou outro setor da mesma, que disputa a mesma clientela e/ou os mesmos recursos eventuais de financiamento, com o a qual não existenenhum tipo de coordenação; Fase 4) Construção do porta-fólio: o porta-fólio deatividadesérepresentado por um gráfico de bolhas, cuja ordenada corresponde ao valor dos segmentos (de 0 a 20), cuja abscissa corresponde à posição competitiva ou grau de controle dos Fatores-Chave de Sucesso (de 0 a 20) e onde o tamanho das bolhas é definido pelo volume de produção das atividades, aqui considerada a média anual. Ele permite refletir sobre as estratégias para os segmentos e o conjunto da 
especialidade e auxilia na elaboração de um plano de ação. 0 porta-fólio é um instrumento de visualização que permite comparar os segmentos, em termos da importância e da posição estratégica dos mesmos, e ter uma visão de conjunto da especialidade; e Fase 5) Plano de ação: visa concretizar os objetivos ou estratégias definidos a partir do porta-fólio. A operacionalização do plano de ação exige a retomada constante da análise estratégica do valor e da posição competitiva, visando aumentar o controle dos fatores-chave de sucesso, para melhorar a posição estratégica geral. Finalmente, a fase da construção de indicadores de monitoramento do plano provê os gestores de informações, permitindo o seu aperfeiçoamento e/ou redirecionamento.

\section{Resultados ediscussão}

A fase de segmentação estratégica definiu coletivamente quatro agrupamentos homogêneos de atividades: Segmento Clínico Dermatológico, Segmento Clínico Otorrinolaringológico, Segmento Laboratório Diagnóstico eSegmento Zoonose. Os segmentos foram definidos a partir dos critérios: patologia, tecnologia e população. A grande importância da fase de segmentação é permitir apreender uma realidade complexa e decompô-la em subconjuntosmaissimples, possibilitando queeste processo seja entendido pelos atores envolvidos. Tal compreensão énecessária para o repasse desta tecnologia para as equipes da instituição.

A análise do valor decada segmento permitiu hierarquizar cada segmento por meio de uma ponderação de critérios e de uma notação relativa, segundo treze critérios. Atribuiu-se um peso relativamente maior aos critérios "M otivação interna", "Sinergias", "Potencial de ensino e pesquisa", e "Capacidade de atração de recursos externos". Os menores pesos foram atribuídos ao critério "Intensidade da Concorrência" - por ser a instituição uma referência nacional einternacional em algumas doenças infecciosas, incluindo a Leishmaniose- e aos critérios "Possibilidade de crescimento", "Investimento tecnológico como barreira à entrada" e "Potencial local", devido à redução, desde 2007, do número de casos novos de Leishmaniose no estado, à necessidade de um investimento tecnológico relativamente baixo em geral e ao expressivo potencial regional e nacional do Laboratório.

Na matriz acima de análise do valor de cada segmento deste Laboratório, destaca-se a valorização de todos os segmentos de uma forma bas- tante homogênea. Tal resultado expressa a importância atribuída a esses segmentos e sua integração à missão institucional. 0 valor um pouco mais baixo atribuído ao Segmento Clínico Otorrinolaringológico expõe uma dificuldade encontrada pelo grupo na produção relacionada à pesquisa. Ressalta-se a previsão de crescimento para a maioria dos segmentos justificada pelo potencial de produção em pesquisa devido a novos doutores formados, com ampliação da capacidade de produção, pela quantidade de dados de pesquisa já coletados e pela possibilidade de estudos multicêntricos. Previu-se uma concorrência de intensidade muito baixa para os segmentos Clínico Otorrinolaringológico e Zoonose, média para o segmento Clínico Dermatológico e alta para o segmento Laboratório Diagnóstico. Registrou-se um baixo investimento tecnológico relativo nos segmentos, sendo muito mais baixo no segmento Clínico Dermatológico e médio no segmento Laboratório Diagnóstico, este em função de equipamentos e materiais de consumo de alto custo. Considerou-se muito alto, em todos os segmentos, o nível de investimento em recursos humanos necessário para alcançar um desempenho diferenciado, sendo esteum fator-chave que diferencia o Laboratório. 0 nível desinergia potencial dos segmentos foi considerado em geral alto, devido à grande colaboração existente com outros Laboratórios em projetos de pesquisa, além de outros setores da instituição (estatística, esterilização, exames de diagnóstico e terapêutica, Pronto Atendimento, Centro Hospitalar, etc.). Ressalta-sea alta motivação da equipe para todos os segmentos. O segmento Clínico Otorrinolaringológico suscitou um grau de motivação relativa um pouco menor devido aos interesses diversos de al guns componentes da equipe, com consequente menor integração com a equipe do Laboratório. De uma maneira geral, os segmentos apresentaram alto potencial de parceria externa tanto em nível nacional quanto internacional para a realização de atividades de pesquisa. Em decorrência da alta qualificação profissional, de um sistema de qualidade implantado e do forte componentede ensino, essas parcerias externas são estabelecidas com finalidades pedagógicas, assistenciais e de fornecimento de insumos, além de atividades de consultorias e apoio desenvolvidas em outros Estados. Todos os segmentos apresentaram um expressivo potencial local, regional enacional de atração e captação de clientela, coerente com a missão da instituição, desenhada para ser referência nacional em Leishmaniose. Do mesmo modo, todos os 
segmentos destacaram-se pelo alto potencial de ensino epesquisa, em sintonia com a missão institucional de desenvolver pesquisa em doenças infecciosas. Com relação à contribuição para o projeto político e para a imagem externa, todos os segmentos valorizam a missão geral e a imagem da instituição. Os quatro segmentos apresentaram boa capacidade de atração de recursos externos que incluem os projetos de pesquisas e as bolsas de estudo.

As Tabelas 1, 2, 3, 4 e 5 mostram a posição competitiva dos diferentes segmentos.

A análise da posição competitiva referiu-se à identificação dos principais Fatores-Chave de Sucesso (FCS) por segmento (Tabelas 2 a 5). Os FCS foram utilizados para avaliar a capacidade de se obter bons resultados em cada um dos segmentos. Foram listados 10 FCS para cada um dos segmentos, exceto o Segmento Zoonose que considerou apenas sete FCS. Distribuíram-se os 100 pontos entre os FCS listados, de modo a ponderar a importância relativa ou peso específico de cada FCS em temos percentuais. O grau de controle por segmento relacionado aos FCS foi obtido por meio deuma notação, considerando como parâmetro, o grau ideal de controle correspondente a um desempenho de alto nível de qualida- de. Para análise dos pontos fortes e fracos do Laboratório de Leishmaniose foram utilizadas as seguintes categorias, considerando o controle dos FCS por segmento: Muito Alto (notas: 17-20), Alto (notas: 13-16), M édio (notas: 8-12), Baixo (notas: 4-7), Muito Baixo (notas: 0-3).

Os pontos fortes correspondem às categorias "Alto" ou "M uito Alto". Nesse sentido, destaca-se 0 alto nível de competitividade do Laboratório de Leishmaniose, avaliado pel o nível de controlealto/ muito alto da maioria dos FCS, em todos os segmentos. De uma forma geral, os principais pontosfortes estão representados pelos seguintesFCS: Trabalho integrado e confiança em equipe; Expertise da equipe; Equipamentos e materiais de consumo de qualidade; Sinergias com outros segmentos; Capacidade de captação e utilização de recursos; Integração das atividades de pesquisa/ ensino/assistência; Articulação com a rede; Sinergias com outros setores do Instituto; e Relação equipe/paciente(tecnologia relacional).

A sinergia entre o segmento Clínico Otorrinolaringológico e outros Laboratórios do instituto foi considerada alta, ocupando grande espaço da agenda, dificultando, inclusive, a marcação de agenda para os próprios pacientes de Leishmaniose. Por outro lado, deve ser aprimo-

\begin{tabular}{|c|c|c|c|c|c|c|}
\hline \multicolumn{2}{|c|}{ Critérios e peso } & \multirow{2}{*}{\multicolumn{2}{|c|}{$\begin{array}{c}\text { Clínico } \\
\text { Dermatológico }\end{array}$}} & \multirow{2}{*}{$\begin{array}{c}\text { Clínico } \\
\text { Otorrinolaringológico } \\
\text { Nota / } 20\end{array}$} & \multirow[t]{2}{*}{$\begin{array}{l}\text { Laboratório } \\
\text { Diagnóstico }\end{array}$} & \multirow[t]{2}{*}{ Zoonose } \\
\hline $\mathrm{N}^{\circ}$ & Critérios de avaliação & & & & & \\
\hline 1 & Possibilidades de crescimento & 5 & 15 & 17 & 18 & 18 \\
\hline 2 & Intensidade da concorrência & 4 & 10 & 17 & 6 & 19 \\
\hline 3 & $\begin{array}{l}\text { Investimento (barreiras à entrada - } \\
\text { Tecnologias) }\end{array}$ & 5 & 2,25 & 4,2 & 6,3 & 3,2 \\
\hline 4 & Investimento (barreiras à entrada - RH ) & 8 & 12,75 & 11,05 & 11,9 & 11,05 \\
\hline 5 & $\begin{array}{l}\text { Sinergias (no nível de competências e } \\
\text { infraestruturas compartilhadas) }\end{array}$ & 10 & 15 & 17 & 15 & 14 \\
\hline 6 & Motivação interna & 11 & 18 & 13 & 18 & 19 \\
\hline 7 & Possibilidades parceria externa & 7 & 16 & 17 & 18 & 19 \\
\hline 8 & Potencial regional & 8 & 17 & 18 & 19 & 19 \\
\hline 9 & Potencial local & 5 & 20 & 20 & 20 & 20 \\
\hline 10 & Potencial de ensino e pesquisa & 10 & 18 & 16 & 18 & 17 \\
\hline 11 & $\begin{array}{l}\text { Contribuição para o projeto político } \\
\text { geral do hospital }\end{array}$ & 9 & 20 & 20 & 20 & 20 \\
\hline 12 & Contribuição para a imagem externa & 8 & 18 & 18 & 18 & 18 \\
\hline \multirow[t]{2}{*}{13} & $\begin{array}{l}\text { Capacidade de atração de recursos } \\
\text { externos }\end{array}$ & 10 & 16 & 13 & 14 & 15 \\
\hline & Valor do Segmento & 100 & 15,88 & 15,52 & 16,11 & 16,48 \\
\hline
\end{tabular}

$*$ Nota $(0-20)+$ Score $=($ Peso $\times$ N ota $) / 100$ 
rada a sinergia com o Centro Hospitalar, pois há dificuldades de internar os pacientes. Essa falta de leitos disponíveis deve-se à priorização de internação de pacientes com AIDS.

Do ponto de vista da equipe, os principais pontos fortes do Laboratório de Leishmaniose referem-se à integração da equipe e dos processos de trabalho, à constituiç̧ão de equipe multiprofissional e ao domínio técnico de diferentes metodologias pela equipe.

Por outro lado, considerando-se o controle dos FCS abaixo de 12 como um ponto fraco, 0

Tabela 2. Posição Competitiva do Segmento Clínico Dermatológico do Laboratório de Leishmaniose, 2009.

\begin{tabular}{|c|c|c|c|c|c|c|c|c|c|c|}
\hline \multirow[b]{2}{*}{$N^{\circ}$} & \multicolumn{4}{|c|}{ Clínico dermatológico } & \multicolumn{2}{|c|}{ Instituição 1} & \multicolumn{2}{|c|}{ Instituição 2} & \multicolumn{2}{|c|}{ Instituição 3} \\
\hline & Fatores Chave de Sucesso & $\begin{array}{c}\text { Peso } \\
\%\end{array}$ & $\begin{array}{l}\text { Nota } \\
/ 20\end{array}$ & $\begin{array}{c}\text { Score } \\
(=p \times n / 100)\end{array}$ & $\begin{array}{l}\text { Nota } \\
/ 20\end{array}$ & $\begin{array}{c}\text { Score } \\
(=p \times n / 100)\end{array}$ & $\begin{array}{l}\text { Nota } \\
/ 20\end{array}$ & $\begin{array}{c}\text { Score } \\
(=p \times n / 100)\end{array}$ & $\begin{array}{l}\text { Nota } \\
/ 20\end{array}$ & $\begin{array}{c}\text { Score } \\
(=p \times n / 100)\end{array}$ \\
\hline 1 & $\begin{array}{l}\text { Trabalho integrado e } \\
\text { confiança em equipe }\end{array}$ & 15 & 18 & 2,7 & 12 & 1,8 & 14 & 2,1 & 16 & 2,4 \\
\hline 2 & Expertise da equipe & 14 & 18 & 2,52 & 17 & 2,38 & 18 & 2,52 & 18 & 2,52 \\
\hline 3 & Estrutura Física & 5 & 10 & 0,5 & 10 & 0,5 & 15 & 0,75 & 10 & 0,5 \\
\hline 4 & $\begin{array}{l}\text { Equipamentos e materiais } \\
\text { de consumo de qualidade }\end{array}$ & 4 & 15 & 0,6 & 13 & 0,52 & 16 & 0,64 & 12 & 0,48 \\
\hline 5 & $\begin{array}{l}\text { Sinergias com outros } \\
\text { segmentos }\end{array}$ & 14 & 18 & 2,52 & 6 & 0,84 & 13 & 1,82 & 13 & 1,82 \\
\hline 6 & $\begin{array}{l}\text { Capacidade de captação e } \\
\text { utilização de recursos }\end{array}$ & 7 & 16 & 1,12 & 17 & 1,19 & 19 & 1,33 & 16 & 1,12 \\
\hline 7 & $\begin{array}{l}\text { Integração das atividades } \\
\text { de pesquisa/ensino/ } \\
\text { assistência }\end{array}$ & 10 & 18 & 1,8 & 16 & 1,6 & 17 & 1,7 & 16 & 1,6 \\
\hline 8 & Articulação com a rede & 6 & 19 & 1,14 & 17 & 1,02 & 17 & 1,02 & 17 & 1,02 \\
\hline 9 & $\begin{array}{l}\text { Sinergias com outros } \\
\text { setores IPEC/FIOCRUZ }\end{array}$ & 7 & 14 & 0,98 & 16 & 1,12 & 16 & 1,12 & 16 & 1,12 \\
\hline 10 & $\begin{array}{l}\text { Quantitativo de } \\
\text { Profissionais }\end{array}$ & 8 & 12 & 0,96 & 9 & 0,72 & 10 & 0,8 & 13 & 1,04 \\
\hline 11 & $\begin{array}{l}\text { Relação equipe/paciente - } \\
\text { Tecnologia Relacional }\end{array}$ & 10 & 17 & 1,7 & 16 & 1,6 & 15 & 1,5 & 16 & 1,6 \\
\hline & Score sobre o segmento & 100 & & 16,54 & & 13,29 & & 15,30 & & 15,22 \\
\hline
\end{tabular}

Tabela 3. Posição Competitiva do Segmento Clínico Otorrinolaringológico do Laboratório de Leishmaniose, 2009.

\begin{tabular}{|c|c|c|c|c|c|c|}
\hline \multicolumn{5}{|c|}{ Clínico Otorrinolaringológico } & \multicolumn{2}{|c|}{ Instituição 1} \\
\hline $\mathrm{N}^{\circ}$ & $\begin{array}{l}\text { Fatores Chave } \\
\text { de Sucesso }\end{array}$ & $\begin{array}{c}\text { Peso } \\
\%\end{array}$ & $\begin{array}{l}\text { Nota } \\
/ 20\end{array}$ & $\begin{array}{c}\text { Score } \\
(=p \times n / 100)\end{array}$ & $\begin{array}{l}\text { Nota } \\
120\end{array}$ & $\begin{array}{c}\text { Score } \\
(=\mathrm{p} \times \mathrm{n} / 100)\end{array}$ \\
\hline 1 & Equipe profissional integrada & 12 & 10 & 1,2 & 10 & 1,2 \\
\hline 2 & Expertise da equipe & 13 & 17 & 2,21 & 16 & 2,08 \\
\hline 3 & Estrutura Física & 12 & 7 & 0,84 & 4 & 0,48 \\
\hline 4 & Equipamentos e materiais de consumo de qualidade & 11 & 15 & 1,65 & 13 & 1,43 \\
\hline 5 & Sinergias com outros segmentos & 11 & 18 & 1,98 & 15 & 1,65 \\
\hline 6 & Capacidade de captação e utilização de recursos & 7 & 16 & 1,12 & 17 & 1,19 \\
\hline 7 & $\begin{array}{l}\text { Integração das atividades de pesquisa/ensino/ } \\
\text { assistência }\end{array}$ & 11 & 18 & 1,98 & 15 & 1,65 \\
\hline 8 & Articulação com a rede & 6 & 16 & 0,96 & 14 & 0,84 \\
\hline 9 & Sinergias com outros setores IPEC/FIOCRUZ & 4 & 14 & 0,56 & 13 & 0,52 \\
\hline 10 & Quantitativo de Profissinais & 7 & 7 & 0,49 & 6 & 0,42 \\
\hline 11 & Relação equipe/paciente - Tecnologia Relacional & 6 & 16 & 0,96 & 15 & 0,9 \\
\hline & Score sobre o segmento & 100 & & 13,95 & & 12,36 \\
\hline
\end{tabular}


Fator 'Estrutura Física' compromete os segmentos Clínico Dermatológico, Clínico Otorrinolaringológico e Laboratório Diagnóstico. A Integração com os Pares de Pesquisa também é menor no segmento Laboratório Diagnóstico. No segmento Otorrinolaringológico, al ntegração da
Equipe também recebeu notação menor. Dentre os segmentos, o de Zoonose apresenta a melhor estrutura física. No segmento Clínico Otorrinolaringológico, destacam-seas limitações no quantitativo de pessoal e no espaço físico para acomodar o recente investimento tecnológico reali-

Tabela 4. Posição Competitiva do Segmento Laboratório Diagnóstico do Laboratório de Leishmaniose, 2009.

\begin{tabular}{|c|c|c|c|c|c|c|c|c|c|c|}
\hline \multicolumn{5}{|c|}{ Laboratório Diagnóstico } & \multicolumn{2}{|c|}{ Instituição 1} & \multicolumn{2}{|c|}{ Instituição 2} & \multicolumn{2}{|c|}{ Instituição 3} \\
\hline $\mathrm{N}^{\circ}$ & Fatores Chave de Sucesso & $\begin{array}{c}\text { Peso } \\
\%\end{array}$ & $\begin{array}{l}\text { Nota } \\
/ 20\end{array}$ & $\begin{array}{c}\text { Score } \\
(=p \times n / 100)\end{array}$ & $\begin{array}{l}\text { Nota } \\
/ 20\end{array}$ & $\begin{array}{c}\text { Score } \\
(=p \times n / 100)\end{array}$ & $\begin{array}{l}\text { Nota } \\
/ 20\end{array}$ & $\begin{array}{c}\text { Score } \\
(=p \times n / 100)\end{array}$ & $\begin{array}{l}\text { Nota } \\
/ 20\end{array}$ & $\begin{array}{c}\text { Score } \\
(=p \times n / 100)\end{array}$ \\
\hline 1 & $\begin{array}{l}\text { Trabalho integrado e } \\
\text { confiança em equipe }\end{array}$ & 15 & 18 & 2,7 & 3 & 0,45 & & 0,6 & 8 & 1,2 \\
\hline 2 & Expertise da equipe & 14 & 17 & 2,38 & 19 & 2,66 & & 2,38 & 19 & 2,66 \\
\hline 3 & Estrutura Física & 8 & 10 & 0,8 & 18 & 1,44 & & 0,96 & 18 & 1,44 \\
\hline 4 & $\begin{array}{l}\text { Equipamentos e materiais } \\
\text { de consumo de qualidade }\end{array}$ & 12 & 15 & 1,8 & 18 & 2,16 & & 1,8 & 18 & 2,16 \\
\hline 5 & $\begin{array}{l}\text { Sinergias com outros } \\
\text { segmentos }\end{array}$ & 14 & 18 & 2,52 & 8 & 1,12 & & 1,12 & 10 & 1,4 \\
\hline 6 & $\begin{array}{l}\text { Capacidade de captação e } \\
\text { utilização de recursos }\end{array}$ & 7 & 15 & 1,05 & 19 & 1,33 & & 1,26 & 19 & 1,33 \\
\hline 7 & $\begin{array}{l}\text { Integração das atividades } \\
\text { de pesquisa/ensino/ } \\
\text { assistência }\end{array}$ & 10 & 18 & 1,8 & 14 & 1,4 & & 1,5 & 15 & 1,5 \\
\hline 8 & Articulação com a rede & 6 & 16 & 0,96 & 17 & 1,02 & & 0,96 & 14 & 0,84 \\
\hline 9 & $\begin{array}{l}\text { Sinergias com outros } \\
\text { setores IPEC/FIOCRUZ }\end{array}$ & 3 & 13 & 0,39 & 8 & 0,24 & & 0,24 & 13 & 0,39 \\
\hline 10 & $\begin{array}{l}\text { Quantitativo de } \\
\text { Profissionais }\end{array}$ & 7 & 10 & 0,7 & 10 & 0,7 & & 0,7 & 10 & 0,7 \\
\hline 11 & $\begin{array}{l}\text { Integração com os pares } \\
\text { de pesquisa (comunidade } \\
\text { científica) }\end{array}$ & 4 & 12 & 0,48 & 17 & 0,68 & & 0,56 & 17 & 0,68 \\
\hline & Score sobre o segmento & 100 & & 15,58 & & 13,20 & & 12,08 & & 14,30 \\
\hline
\end{tabular}

Tabela 5. Posição Competitiva do Segmento Zoonose do Laboratório de Leishmaniose, 2009.

\begin{tabular}{|c|c|c|c|c|c|c|c|c|}
\hline \multirow[b]{2}{*}{$\mathrm{N}^{\circ}$} & \multicolumn{2}{|l|}{ Zoonoze } & \multirow[b]{2}{*}{$\begin{array}{c}\text { Nota } \\
/ 20\end{array}$} & \multirow[b]{2}{*}{$\begin{array}{c}\text { Score } \\
(=p \times n / 100)\end{array}$} & \multicolumn{2}{|c|}{ Instituição 1} & \multicolumn{2}{|c|}{ Instituição 2} \\
\hline & Fatores Chave de Sucesso & $\begin{array}{l}\text { Peso } \\
\%\end{array}$ & & & $\begin{array}{l}\text { Nota } \\
/ 20\end{array}$ & $\begin{array}{c}\text { Score } \\
(=p \times n / 100)\end{array}$ & $\begin{array}{l}\text { Nota } \\
/ 20\end{array}$ & $\begin{array}{c}\text { Score } \\
(=p \times n / 100)\end{array}$ \\
\hline 1 & Trabalho integrado e confiança em equipe & 14 & 18 & 2,52 & 4 & 0,56 & & 0,56 \\
\hline 2 & Expertise da equipe & 19 & 16 & 3,04 & 16 & 3,04 & & 0,57 \\
\hline 3 & Estrutura Física & 7 & 16 & 1,12 & 20 & 1,4 & & 0,7 \\
\hline 4 & $\begin{array}{l}\text { Equipamentos e materiais de consumo de } \\
\text { qualidade }\end{array}$ & 6 & 16 & 0,96 & 18 & 1,08 & & 0,6 \\
\hline 5 & $\begin{array}{l}\text { Sinergias com outros segmentos, } \\
\text { principalmente Laloratório }\end{array}$ & 198 & 20 & 3,8 & 15 & 2,85 & & 0,57 \\
\hline 6 & Capacidade de captação e utilização de recursos & 14 & 13 & 1,82 & 16 & 2,24 & & 0,42 \\
\hline 7 & Quantitativo de Profissionais & 7 & 7 & 0,49 & 7 & 0,49 & & 0,49 \\
\hline 8 & $\begin{array}{l}\text { Integração das atividades de pesquisa/ensino/ } \\
\text { assistência }\end{array}$ & 14 & 17 & 2,38 & 14 & 1,96 & & 1,4 \\
\hline & Score sobre o segmento & 100 & & 16,13 & & 13,62 & & 5,31 \\
\hline
\end{tabular}


zado, embora exista a perspectiva de mudança da estrutura física na proposta do Plano Diretor do instituto.

Muitas atividades do Laboratório de Leishmaniose são desenvolvidas por alunos. Nesse sentido, com relação ao segmento Laboratório Diagnóstico, há deficiência de profissionais contratados para a realização de sorologias diagnósticas, fragilizando essa atividade.

Dos seis concorrentes/parceiros do Laboratório de Leishmaniose identificados na fase da Análise do Existente, selecionaram-se os principais de cada segmento, sendo apontados: três concorrentes/parceiros para o segmento Clínico Dermatológico, um para o Clínico Otorrinolaringológico, três para o Laboratório Diagnóstico e dois para o segmento de Zoonose. Em relação a esses concorrentes/parceiros listados, 0 Laboratório de Leishmaniose apresentou melhor competitividade em todos os segmentos, em alguns casos com nota próxima aos concorrentes, em outros, sobressaindo-se.

A análise revela uma excelentecompetitividade do Laboratório de Leishmaniose, porém sugere a necesssidade deum esforço deinvestimento deforma geral na estrutura física do Laboratório, na integração da equipe do segmento Clínico Otorrinolaringológico e na integração com os pares de pesquisa do segmento Laboratório Diagnóstico.

0 porta-fólio mostra, sinteticamente, o valor concorrencial e o risco estratégico de cada segmento e possibilita uma visualização de conjunto do laboratório. Em função da complexidade da missão institucional onde as atividades de pesquisa sobrepõem-se, sendo consideradas 0 "carro-chefe", a partir das quais a assistência se subordina, deparamo-nos com o desafio de criar indicadores que expressem essa complexidade. Os dados de produção das atividades desenvolvidas em 2008 no Laboratório de Leishmaniose foram separados por segmentos e por tipo de produção (assistencial, de pesquisa e de ensino). Ressalta-se a importância de estabelecer pesos diferenciados para os diferentes tipos de produção. Nesse sentido, foram construídos dois porta-fólios de atividades, sendo um referente à produção assistencial eoutro com dados de produção de ensino e pesquisa.

Com relação aos indicadores de produção assistencial, o volume de produção foi calculado pela somatória simples, independente da atividade (consulta, procedimento ou exame) por se entender que esse modo expressa a realidade assistencial dos segmentos. Para a construção do porta-fólio de ensino e pesquisa foram estabelecidos pesos diferenciados para os diversos tipos de indicadores de produção, divididos em sete categorias com os respectivos pesos: elaboração de artigos científicos; participação em congressos, captação de recursos, orientações científicas, participação em bancas científicas, coordenação de cursos, participação em cursos da instituição como docente. Cada categoria foi dividida em subcategorias com atribuição de pesos segundo consenso pela equipe após uma discussão sobre critérios de avaliação de produção científica e da pós-graduação apoiada em literaturara-19 ${ }^{16}$ obre o tema e na experiência de coordenação de programa de pós-graduação de dois participantes. A categoria Artigos Científicos foi pontuada segundo o nível de classificação dos periódicos na avaliação da Capes (Coordenação deA perféçoamento de Pessoal de Nível Superior), através do chamado Qualis-Capes. Este aferea qualidade dos artigosa partir da análise da qualidade dos veículos de divulgação, ou seja, periódicos científicos. A classificação de periódicos é real izada pelas áreas de avaliação e é atualizada anualmente. Os estratos indicativos da qualidade são $A 1$, o mais alto; $A 2 ; B 1$; B2; B3; B4; B5 e C. Para efeitos da análise para a produção do Laboratório de Leischmaniose consideramos a produção de artigos a partir de B2, desconsiderando a produção de menor indexação devido ao nível de exigência cada vez maior na avaliação dos programas, dos docentes e conse quentemente da competitividade dos doutores para captação de recursos de pesquisa. A categoria Captação de Recursos foi classificada segundo o valor monetário dos recursos captados. Alguns exemplos: até 10.000 reais equivaleu a 20 pontos; de 40.000 a 100.000 a 50 pontos, acima de um milhão a 200 pontos, entre outros.

0 porta-fólio assistencial (Figura 1) mostra que nenhum segmento está fora do quadrante superior direito, evidenciando que o Laboratório, de forma geral, encontra-se bem adaptado à missão da instituição e com pouco risco estraté gico. 0 volume de produção assistencial é relativamente alto, principalmente dos segmentos Laboratório Diagnóstico e Clínico Otorrinolaringológico.

0 porta-fólio de ensino e pesquisa, (Figura 2) embora expresse a adequação do Laboratório ao perfil desejado para a instituição, apresenta maior diversidade entre os segmentos com destaque para o segmento Clínico Dermatológico que concentra a produção de ensino e pesquisa. O segmento Clínico Otorrinolaringológico reve la a menor produção. Tal fato aponta para a ne cessidade de um maior investimento em ações 
voltadas para aumentar a produção em ensino e pesquisa particularmente no segmento Clínico Otorrinolaringológico. Sugeriu-se aumentar a

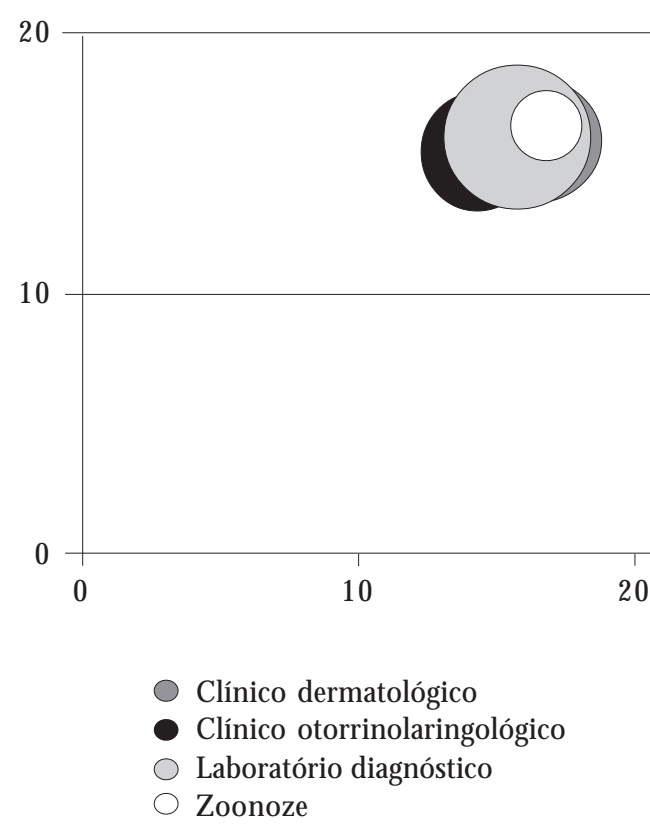

Figura 1. Porta-fólio Assistencial do Laboratório de Leishmaniose, 2009.

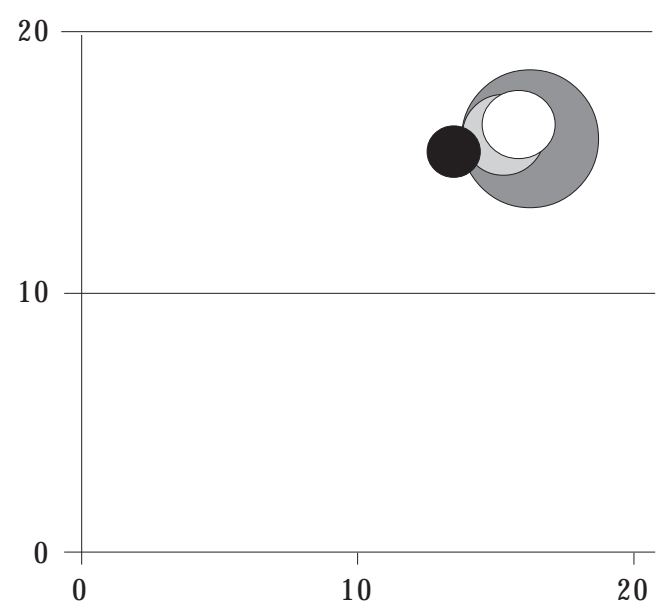

Clínico dermatológico

Clínico otorrinolaringológico

Laboratório diagnóstico

Zoonoze

Figura 2. Porta-fólio de Ensino e Pesquisa do Laboratório de Leishmaniose, 2009. produção científica, principalmentea publicação nas melhores revistas, e desconcentrar a captação de recursos por meio de outros doutores, para além do segmento Clínico Dermatológico.

$M$ ais importante que a comparação sobre as bases mais ou menos 'objetivas' dos dados mostrados nos porta-fólios, esta discussão insere-se como elemento comunicativo da negociação do plano. Assim, esta fase tem o potencial de expressar o processo de cogestão, onde os diferentes atores envolvidos são incluídos na formulação de políticas, no processo de trabalho e na avaliação dos resultados e impactos da produção organizacional. É importante destacar que toda a equipe do Laboratório, entre chefias, pesquisadores e estudantes de pós-graduação, participou de todo processo.

As estratégias foram inicialmente construídas a partir da apropriação dos FCS e da identificação dos pontos fracos identificados. Com relação à estrutura física, embora se reconheçam problemas estruturais que precisam ser resolvidos, a equipe identifica essa problemática como uma deficiência crônica da instituição. Como a demanda assistencial tem diminuído no estado, avalia-se que não há possibilidade de reivindicar muitas alterações na estrutura atual.

Sobre o fator-chaverecursos humanos, apontou-se a necessidade de investir em capacitação e experiência profissional ena captação externa de profissionais para todos os segmentos ( por meio de Concurso Público ou outros). Em relação à demanda por um maior quantitativo de profissionais, apontou-se a necessidade de contratar mais um médico clínico dermatologista, um mé dico clínico otorrinolaringologista, um veterinário, quatro profissionais de nível médio sendo três para o segmento do Laboratório Diagnóstico e um para o segmento de Zoonose. Além disso, há necessidade de se criar quadro para contratação de um fonoaudiólogo para operacionalizar os equipamentos de recente aquisição.

Para o aperfeiçoamento das ações assistenciais e para aumentar o impacto em pesquisa, indicaram-se ações de incorporação tecnológica, principalmente no segmento do Laboratório Diagnóstico, e ações de comunicação e negociação relacionadas ao estabelecimento de sinergias internas com outros laboratórios (particularmente com a anatomia patológica), e parcerias externas de integração com os pares de pesquisa.

A análise dos resultados sugere a presença no interior da equipe deal gumas características que correspondem aos impactos culturais buscados por meio da implementação da Démarche. 0 
tempo cronológico da experiência de aplicação desteenfoquenuma instituição de pesquisa pode ser considerado insuficientepara sefazer afirmações sobre transformações em relação à cultura organizacional. Nesta medida, ponderamos que a presença dessas características corresponde, em parte, a uma evolução cultural prévia que facilitou ou que foi coerente com a aplicação da lógica do enfoque. Porém, consideramos que esta expe riência contribuiu para potencialização destestraços emergentes. Esses traços permitiriam contrabalançar configurações típicas das organizações profissionais4 ${ }^{4}$, decorrentes da alta autonomia dos centros operacionais e da grande diferenciação entre equipes, serviços, ecomponentes organizacionais. A análise desses traços culturais nos permite afirmar o potencial comunicativo da D émarcheno sentido de considerar a possibilidade do entendimento, da cooperação e da ne gociação, a partir de uma ação prática estratégica de redefinição da missão institucional numa perspectiva de rede coordenada de serviços.

Outraquestão importantenas experiências de gestão para mudança é a qualidade das lideranças, destacando-se os traços de competência intersubjetiva e comunicativa ${ }^{20}$. Destaca-se a participação da chefia do Laboratório de Leishmaniose, juntamente com sua equipe de pesquisadores e estudantes, em todas as reuniões. 0 apoio e a capacidade de motivação dessa liderança no sentido de convocar e garantir a participação de todos os atores do Laboratório foi uma estratégia muito interessante, pois permitiu a apropriação do método por todos, estimulou a reflexão coletiva e permitiu compartilhar responsabilidades. Nesse contexto, de uma forma geral, observou-se que a experiência baseou-se no diálogo entre a chefia do Laboratório e os profissionais da ponta, apoiados pela lógica da direção estratégica do Instituto para a formalização das análises, avaliações e estratégias necessárias à elaboração de um plano comum. N esse contexto, reconhecemos uma boa incorporação do raciocínio gerencial estratégico e de uma visão de conjunto do Laboratório pela equipe, assim como a presença de uma equipemultiprofissional do tipo integração ${ }^{21}$, quefoi, inclusive, definidaoperacionalmentecomo um Fator Chave de Sucesso (FCS) em todos os segmentos, sendo reconhecida como outro traço cultural emergente. Para Peduzzi ${ }^{22}$, a integração, conceito baseado em Habermas, constitui-se, "por meio da relação recíproca, de dupla mão, entrea articulação das ações executadas pelos distintos profissionais ea interação desses profissionais, visto quea comunicação éo veículo quepos- sibilita a própria articulação". Este estudo possibilita observar que essa articulação foi assegurada tanto pelo potencial comunicativo da Démarche, capaz de fomentar a inclusão dos sujeitos nos arranjos, processos e dispositivos de gestão, como pela capacidade da equipe que mostrou desde o início uma cultura de diálogo eresponsabilização coletiva. N esse sentido, é inegável que a concretização desta possibilidade dependeu da capacidade de mobilização e de comunicação do coletivo, articulada ao grau de compromisso de monstrado pelas lideranças locais.

Além da maior comunicação entre a direção estratégica e os profissionais da ponta e da busca de um enfoque multiprofissional, por meio de articulação de ações e integração dos profisssionais (rede interna), destaca-se a maior abertura para o externo (rede externa), concretizada pelo reconhecimento e exploração das parcerias e sinergias?. A visão do ambiente como recurso e a perspectiva de estabelecer redes configuram outro traço cultural identificado na equipe detrabaIho. Podemos afirmar que uma equipe multiprofissional do tipo integraçãa ${ }^{22}$ e a constituição de redes representam ligações que pressupõem confiança na ação de compartillhamento de objetivos e projetos ${ }^{23,24}$. No contexto desses resultados alcançados, ressalta-seo potencial comunicativo da Démarcheno desenvolvimento de relações deconfiança, sob a ótica da linguagem de Echeverria ${ }^{25}$, segundo a qual, a confiança ou a desconfiança resulta do nível de cumprimento dos compromissos inerentes aos atos de fala proferidos relacionados com a competência, a sinceridade e a responsabilidade dos atores envolvidos. Esta re lação de confiança é observada também em relação aos pacientes atendidos pelo Laboratório e que participam de diferentes fases de pesquisa, revelando um vínculo forte entre os profissionais e os usuários, condição preconizada pela política de humanização do M inistério de Saúde ${ }^{6,26}$. Esse vínculo, queaparececomo "tecnologia relacional", é considerado um FCS importante dos dois segmentos mais assistenciais do Laboratório.

Outro aspecto importante a ser apontado, em função da complexidade da instituição em pauta, que abrange atividades assistenciais, de ensino e pesquisa, foi o desafio de superação metodológica no sentido de se criar indicadores mistos de produção. A utilização destes novos indicadores permitiu identificar um novo traço cultural que diz respeito ao desenvolvimento de uma cultura de avaliação. Com essa experiência, reitera-se ser uma condição importante para a implementação da lógica de avaliação, a existên- 
cia de um bom sistema de informação institucional e um cuidado com o registro da produção científica eassistencial.

Este estudo nos permite sistematizar al guns desafios relacionados à aplicação da démarche em uma instituição onde se destaca a pesquisa em sua missão. Em primeiro lugar, foi necessário reforçar o peso do critério potencial de ensino e pesquisa na análise do valor dos segmentos. Houve, ainda, como afirmado acima, uma adaptação dos indicadores de produção que permitiu a construção de um porta-fólio específico de ensino epesquisa. Evidenciou-sea existência deum bom sistema de informações sobre a produção em geral, o que consideramos que se deve principalmente ao ambiente cultural de pesquisa que exige o registro minucioso de todos os dados, inclusive assistenciais para o bom andamento da própria pesquisa. Além disso, a cobrança ea prestação de contas motivada pelas agências de financiamento também contribuem para este efeito. Outro desafio inerente ao fato da aplicação deste enfoque numa instituição onde a pesquisa prepondera refere-se à segmentação estratégica. No caso desta aplicação, a segmentação ainda obedeceu mais a critérios assistenciais: população, tecnologia e patologia, mas poderia ter obe- decido a outra lógica típica da pesquisa. Em outras experiências ${ }^{27}$ a segmentação foi feita a partir decritérios mais específicos relacionados ao tipo de estudo epidemiológico ou clínico, resultando em segmentos como: ensaios clínicos profiláticos, ensaios clínicos terapêuticos, estudos de coorte de mulheres, dentre outros. No caso do laboratório deleishmaniose, o grupo preferiu uma segmentação assistencial, revelando tal vez o peso que a assistência tem no mesmo. Além da segmentação e da avaliação dos segmentos que é sempre um desafio, outro, apontado no plano de atividades refere-se à cooperação entre grupos de pesquisa, às vezes mais complexa que entre instituições assistenciais, com parcerias em determinados projetos ou suas fases e que impactam a qualidade da própria assistência. Esteé um aspecto que deve ser mais explorado em outras experiências.

Finalmente, reafirma-se que esta experiência de aplicação da Démarche Estratégica, por operar indiretamente como reforço dos traços da nova cultura que emerge, revelou o potencial do método em pauta, para efetivar políticas, processos e recursos de forma comunicativa e democrática, o que contribui para a operacionalização das diretrizes do SUS.

\section{Colaboradores}

E Artmann, M AC Andrade e FJU Rivera participaram de todas as fases da pesquisa e da redação do artigo. 


\section{Referências}

1. Cecílio LCO. A modernização gerencial dos hospitais públicos: o difícil exercício da mudança. Rev Adm Pública 1997; 31(3):36-47.

2. Peduzzi M. Equipe multiprofissional de saúde: conceito e tipologia. Rev Saude Publica 2001; 35(1):103109.

3. Crémadez $M$, Grateau $F$. Le management stratégique hospitalier. Paris: InterÉditions; 1997.

4. M intzberg $\mathrm{H}$. Structure et Dynamique des Organisations. Paris: Les Éditions d'Organisation; 1982.

5. Rivera FJU. Cultura e liderança comunicativa. In Rivera FJU, organizador. Análise Estratégica em saúde e Gestão pela Escuta. Rio de Janeiro: Fundação Oswaldo Cruz; 2003. p. 185-218.

6. Artmann E, Rivera FJU. Humanização no atendimento e gestão comunicativa. In: Deslandes SF, organizadora. Humanização dos cuidados em saúde: conceitos, dilemas e práticas. Rio de Janeiro: Fiocruz; 2006. p. 205-231.

7. Artmann E. Enfoque da Démarche Stratégique na Gestão Hospitalar. In: M inayo MCS, Deslandes SF, organizadoras. Caminhos do Pensamento: epistemologia e método. Rio de Janeiro: Editora Fiocruz; 2002. p. 277-323

8. Lopes CM B. 'Démarche Stratégique': uma metodologia a ser proposta para a gestão hospitalar brasileira [dissertação]. Rio de Janeiro: Escola Nacional de Saúde Pública; 1997.

9. Souza RM P. A démarche estratégica: uma abordagem teórico-metodológica [dissertação]. Rio de Janeiro: Escola Nacional de Saúde Pública; 1997.

10. Favacho JCP. Gestão estratégica da maternidade da Fundação Santa Casa de M isericórdia do Pará: a Dé marche Stratégique em questão [dissertação]. Rio de Janeiro: Escola Nacional de Saúde Pública; 2001.

11. Artmann E. Démarche Stratégique (gestão estratégica hospitalar): um enfoque que busca a mudança através da comunicação e solidariedade em rede [tese] Campinas: Faculdade de Ciências M édicas; 2002.

12. Rivera FJU, Artmann E. Planejamento e gestão em saúde: flexibilidade metodológica e agir comunicativo. In: Rivera FJU, organizadores. Análise Estratégica em saúde e Gestão pela Escuta. Rio de Janeiro: Fiocruz; 2003. p. 17-35.

13. Artmann E, Rivera FJU. A Démarche Stratégique (gestão estratégica hospitalar): um instrumento de coordenação da prática hospitalar baseado nos custos de oportunidade e na solidariedade. Cien Saude Colet 2003; 8(2):479-499.

14. Andrade M AC, Artmann E. Démarche Estratégica numa unidade materno infantil: um instrumento de gestão hospitalar. Rev Saude Publica 2009; 43(1): 105-114.

15. Rivera FJU. A démarche estratégica: a metodologia de gestão do Centro Hospitalar Universitário de Lille, França. Cad Saude Publica 1997; 13(1):73-80.

16. Brasil. Ministério da Educação. Coordenação de Aperfeiçoamento de Pessoal de Nível Superior. Plano Nacional de Pós-graduação - PN PG 2005-2010. Brasília: Capes; 2004. [acessado 2009 Jun 23]. Disponível em: http://www.capes.gov.br/images/stories /download/editais/PN PG 2005 2010.pd
17. Guimarães JA. Pesquisa em saúde no Brasil: contexto e desafios. Rev Saude Publica 2006; 40(N Esp.):3-10.

18. Guimarães JA. A pesquisa médica e biomédica no Brasil: comparações com o desempenho científico brasileiro e mundial. Cien Saude Colet 2004; 9(2):303327

19. Barros AJD. Produção científica em saúde no Brasil: perfil dos periódicos e avaliação pela CAPES. Rev Saude Publica 2006; 40(N. Esp.):43-49.

20. Rivera FJU, Artmann E. A liderança como intersubjetividade lingüística. Interface - Comun Saúde Educ 2006; 10(20):411-422.

21. Peduzzi M. Equipe multiprofissional de saúde: conceito e tipologia. Rev Saude Publica 2001; 35(1):103109.

22. Peduzzi M. Trabalho em equipe de saúde no horizonte normativo da integralidade, do cuidado e da democratização das relações de trabalho. In: Pinheiro R, Barros MEB, M attos RA, organizadores. Trabalho em equipe sob o eixo da integralidade: valores, saberes e práticas. Rio de Janeiro: Abrasco; 2007. p. 161-177.

23. Rovere $M$. Redes en salud: un nuevo paradigma para el abordaje de las organizaciones y la comunidad. Rosario: Ed. Secretaría de Salud Pública/ AM R, Instituto Lazarte (reimpresión); 1999 [acessado 2011 fev 24]. Disponível em: http://www. ilazarte.com.ar/mt-static/blog/archives/M ario\% 20Rovere $\% 20$ - $\% 20$ libro\%20\%20Redes $\% 20$ en $\%$ 20Salud.pdf

24. Fleury S, Ouverney AM. Redes de política: emergência, conceituação e gestão. In: Fleury S, Ouverney AM, organizadores. Gestão de redes: estratégia de regionalização da política de saúde. Rio de Janeiro: FGV; 2007. p. 09-35.

25. Echeverria R. Confiança: viga mestra da empresa de futuro. Reflexão São Paulo [periódico na internet]. 2008 [acessado 2011 fev 24]; 3(7): [26 p.]. Disponível em: http://www1.ethos.org.br/EthosWeb/ arquivo/0-A-1f4reflexao\%2007.pdf

26. Deslandes S, Mitre RM A. Processo comunicativo e humanização em saúde. Interface Comun Saúde Educ 2009; 13(Supl. 1):641-649.

27. Artmann E, Rivera FJU, Andrade MAC. Relatório do Projeto de Pesquisa Humanização dos Serviços de Saúde: gestão estratégica no trabalho, produção de saúde e análise cultural. Rio de Janeiro; 2010.

Apresentado em 13/07/2011

Aprovado em 10/10/2011

Versão final apresentada em 21/10/2011 